\title{
High Frequency Ultrasound Signal Statistics from Mouse Mammary Tissue During Involution
}

\author{
A. S. Tunis \\ University of Toronto \\ D. Spurell \\ Ontario Cancer Institute \\ D. McAlduff \\ Ontario Cancer Institute
}

\section{A. Giles}

Ontario Cancer Institute

\section{Hariri}

University of Toronto M. D., Czarnota, G. J., \& Kolios, M. C. (2004). High frequency ultrasound signal statistics from mouse mammary tissue during involution. IEEE Ultrasonics Symposium, 1, 768-771.

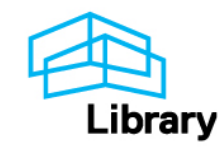




\title{
High Frequency Ultrasound Signal Statistics From Mouse Mammary Tissue During Involution
}

\author{
A.S. Tunis ${ }^{* \dagger}$, D. Spurrell ${ }^{\dagger}$, D. McAlduff ${ }^{\dagger}$, A. Giles ${ }^{\dagger}$, M. Hariri ${ }^{* \dagger}$,

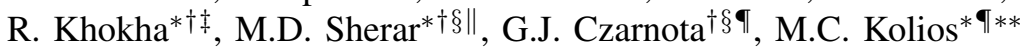 \\ Departments of ${ }^{*}$ Medical Biophysics, ${ }^{\S}$ Radiation Oncology and $\ddagger$ Laboratory Medicine and Pathobiology, \\ University of Toronto, Toronto, Ontario, Canada \\ ${ }^{\dagger}$ Ontario Cancer Institute, University Health Network, Toronto, Ontario, Canada \\ TDepartment of Mathematics, Physics and Computer Science, Ryerson University, Toronto, Ontario, Canada \\ " London Regional Cancer Centre and Department of Oncology, University of Western Ontario, London, Ontario, Canada \\ **Corresponding author: mkolios@ryerson.ca
}

\begin{abstract}
We investigate the use of signal envelope statistics to monitor the tissue restructuring process during mouse mammary tissue involution. Using a $f / 3$ transducer operating at a centre frequency of $20 \mathrm{M} \mathrm{Hz}$, ultrasound backscatter data were collected from mouse mammary tissue following removal of the litter. The signal envelope statistics were examined by fitting the Rayleigh and generalized gamma distributions. The goodness of fit of the distributions was evaluated by the Kolmogorov-Smirnov test.

The goodness of fit of the Rayleigh distribution increased to a maximum at day 3 of involution, roughly corresponding to the peak of apoptosis in the tissue. Similarly, the fit parameters of the generalized gamma distribution indicate that at day 3 the distribution is most Rayleigh like. It is believed that the condensation of the nucleus and reorganization of the tissue structure produced these changes in the statistics of the signal envelope. The results demonstrate that high frequency ultrasound signal envelope statistics can be applied to monitor changes to tissue structure in vivo.
\end{abstract}

\section{INTRODUCTION}

It has previously been demonstrated that high frequency ultrasound (HFUS) backscatter signals show large changes in response to the structural changes that occur in cells during cell death. Specifically an increase in integrated backscatter (IB) and changes to the shape of the frequency spectrum have been observed during the treatment of cells with DNA intercolating agents [1]-[3]. While the IB and frequency spectra can be used to monitor changes in an in vitro model, the numerous changes that may occur to both cell and tissue structure in an in vivo model raise new challenges. As a complementary technique we investigate the use of envelope statistics analysis for monitoring structural changes in cells [4]. Changes in cell structure affect the distribution of the signal amplitudes, and this can be quantified by examining fits of theoretical probability density functions (PDFs) to the backscattered HFUS signals.

The use of envelope statistics analysis for ultrasonic tissue classification has previously been demonstrated in vivo for a number of clinical applications. At lower frequencies Shankar et al. [5], [6] demonstrated its application to classifying breast tumours, and Hao et al. [7], [8] demonstrated its use for differentiating normal and infarcted myocardium. More recently
Raju et al. [9] demonstrated envelope statistics analysis with HFUS for differentiating between skin types.

We present an investigation of the use of this technique to monitor structural changes during apoptotic cell death and tissue remodelling using the in vivo model of mouse mammary tissue during the period of involution following removal of the litter. The tissue restructuring during this process involves a large reduction in the volume of the mammary tissue, and elimination of cells by apoptosis [10].

\section{THEORY}

The HFUS backscatter signal from tissues may be represented as a sum of backscattered signals from individual scatterers within the resolution volume of the transducer (Equation 1).

$$
r e^{j \Psi}=\sum_{i=1}^{n} r_{i} e^{j \theta_{i}}
$$

Each scattering source contributes an amplitude $r_{i}$ and a phase $\theta_{i}$, to give a total amplitude $r$ and phase $\Psi$. The phase and amplitude contributed by each scatterer is dependent on the acoustic properties of that scatterer. In tissue these properties vary from one scatterer to another, as such the amplitude and phase contributions will have some statistical distribution. This produces a total amplitude and phase that has a statistical nature, varying throughout the tissue. The stochastic fluctuations of the amplitude produce the characteristic speckle pattern observed in ultrasound B-scan images.

There are a number of different theoretical PDFs that have been applied to model the statistics of the signal amplitude. One of the simplest models is the Rayleigh PDF, which models the signal resulting from a large number of point sources at random locations (Equation 2) [11].

$$
\begin{gathered}
p(r)=\frac{r}{\sigma^{2}} e^{\frac{-r^{2}}{2 \sigma^{2}}} \\
r \geq 0 ; \sigma>0
\end{gathered}
$$

While useful as a first approximation, the Rayleigh PDF does not provide a good fit to signals from tissues. To date 
our experimental evidence indicates that the nucleus is the primary scatterer in the cell [12], [13]. There are a large number of cells (approximately 600) in the resolution volume of the transducer used. However, the size of the nucleus is approximately $10 \mu \mathrm{m}$ in diameter, which is smaller, but of the same order of magnitude as the wavelength $(75 \mu \mathrm{m}$ at $20 \mathrm{MHz}$ ). And finally the nuclei are not at completely random positions within a sample of tissue. The limited validity of the assumptions results in the poor fit provided by the Rayleigh PDF.

Recently the generalized gamma (GG) PDF [14] was proposed by Raju et al. [9] as a model for backscattered HFUS from tissue. The GG PDF is more flexible and can account for a wider range of scattering interactions, such as organization of the positions of the scatterers. It is a three parameter distribution, allowing it to provide a very good fit to measured signals (Equation 3).

$$
\begin{gathered}
p(r)=\frac{c r^{c v-1}}{a^{c v} \Gamma(v)} e^{-\left(\frac{r}{a}\right)^{c}} \\
r \geq 0 ; a \geq 0 ; v \geq 0 ; c \geq 0
\end{gathered}
$$

The GG PDF is very similar to the generalized Nakagami PDF proposed by Shankar [15]. As the GG PDF is capable of accommodating a wider range of scattering conditions, it is also capable of representing a number of other distributions as special cases. When $c=2$ and $v=1$ the GG PDF becomes the Rayleigh PDF, with the $a$ parameter in the GG PDF equal to $\sigma$ of the Rayleigh PDF.

\section{METHODS}

For seven days following the weaning of the litter the mammary tissue of four mice was imaged daily. During the procedure the animals were anaesthetized and placed in a restraining device in a heated water bath. The restraining device was designed to support the animal's head out of the water, while keeping the mammary tissue below the water such that the water was used as a coupling medium to the transducer (Figure 1).

Data were acquired using a VS-40b HFUS scanner from VisualSonics with an $f / 3$ transducer pulsed at a centre frequency of $20 \mathrm{MHz}$ (VisualSonics Inc., Toronto, Canada). The focus of the transducer was positioned $1.5 \mathrm{~mm}$ below the surface of the pellet. B-scan images and RF data from approximately 200 locations separated by at least one beamwidth were acquired from several imaging planes within the mammary tissue. The lymph node (hypoechoic region in Figure 2) was used as a reference point to ensure data were collected from the same region of tissue every day.

Using custom software written in JAVA (JDK 1.4.1, Sun Microsystems, Inc., Santa Clara, CA, USA), RF data were extracted from a relatively homogeneous region of interest (ROI) within the mammary tissue. The ROI was selected to be from $0.5 \mathrm{~mm}$ above the focus to $0.5 \mathrm{~mm}$ below the focus. This is well within the depth of field $(2.5 \mathrm{~mm})$ such that the decrease in signal over the $1 \mathrm{~mm}$ ROI would be primarily due

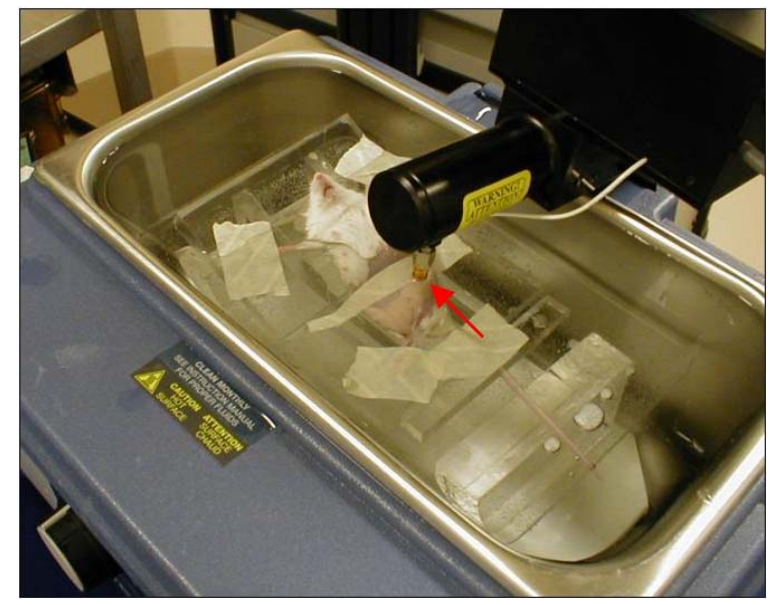

Fig. 1. Setup to immobilize the mammary tissue of the mouse in the heated water bath which was used as a coupling medium. The transducer (indicated by the arrow) was scanned linearly across the mammary tissue.

to attenuation. To correct for this, the attenuation in $d B / m m$ was determined as the decrease in mean signal envelope over the $1 \mathrm{~mm}$ depth. Using this attenuation value the data were corrected for attenuation by applying depth dependant amplification.

Based on the attenuation corrected RF data from the ROI, PDF fit parameters for the Rayleigh and GG PDFs were determined using the maximum likelihood estimation (MLE) routine in Matlab (Version 6.1, The MathWorks Inc., Natick, MA, USA). The goodness of fit of the two PDFs were evaluated by the Kolmogorov-Smirnov (KS) goodness of fit test [16].

\section{RESUlts}

Throughout the period of involution there was a significant reduction in the volume of the mammary tissue. This is apparent in the digital photos and B-scan images of the mammary tissue (Figure 2). While changes to the structure of the cells are apparent in the histology, the changes to the tissue composition are much more dramatic. The tissue composition changes from predominantly epithelial cells at day 0 to mostly fat by day 6 of involution. As a result the most prominent changes in the B-scan images are from the restructuring and reduced volume of the tissue. The changes in intensity within the mammary tissue that would be expected due to the structural changes occurring within the cells are difficult to observe due to the large changes in tissue structure.

The GG PDF provided a much better fit than the Rayleigh PDF, with KS goodness of fit values almost one order of magnitude less for the GG than the Rayleigh. Furthermore the KS values indicate that at day 2 of involution the signal tends towards Rayleigh statistics, while at later times the statistics become increasingly non-Rayleigh (Figure 3 ). This is close to the peak of apoptotic cell death expected around day 3 of involution [10]. 


\section{Day 0}
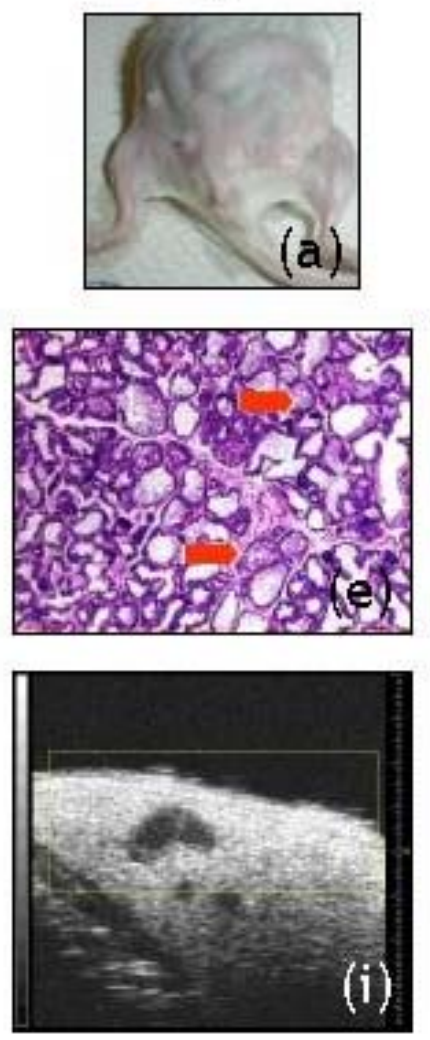

Day 3
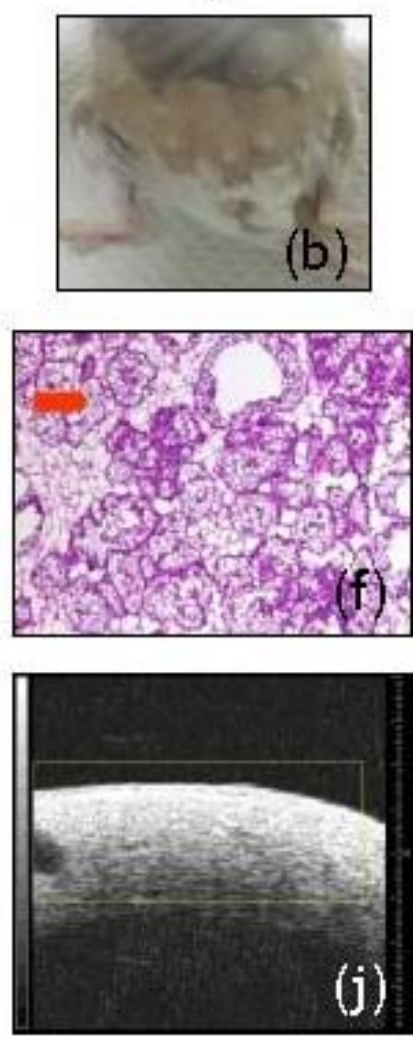

Day 4
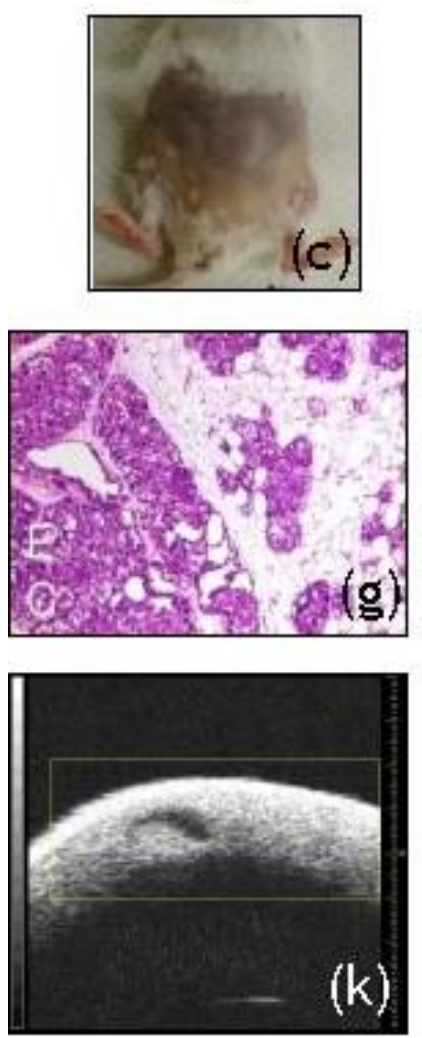

Day 6
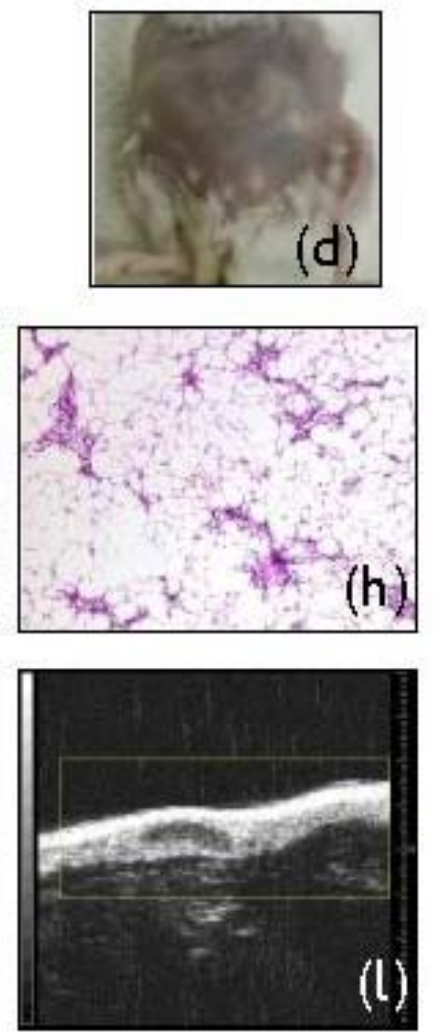

Fig. 2. Digital photographs (a-d) and H\&E histology (e-h) of mouse mammary tissue showing large changes to the tissue structure and composition throughout the involution period. Images of the histological sections were taken at $40 \times$ magnification, the arrows indicate alveolar ducts. B-scan images (Field of View is $8 \mathrm{~mm} \times 8 \mathrm{~mm}$ ) of the same tumour with a $20 \mathrm{MHz} / \mathrm{f} / 3$ transducer also display the structural changes clearly (i-1). The lymph node (hypoechoic region) was used as a reference to ensure imaging was performed of the same region each day.

The parameters of the GG PDF show good sensitivity to changes in tissue structure. The GG a parameter reaches a maximum at day 1 of involution, decreasing sharply afterwards (Figure 4). As the GG $c$ and $v$ parameters approach the special case of $c=2$ and $v=1$ the GG PDF becomes similar to the Rayleigh PDF (Equations $2 \& 3$ ). The GG $c$ and $v$ parameters approach these values between days 1-3, corresponding to the maximum of the Rayleigh goodness of fit at day 2 (Figures $3 \& 4)$. This coincides with the peak of apoptotic cell death around day 3 of involution [10].

\section{DISCUSSION}

HFUS signals were collected from mouse mammary tissue during the first six days of involution. The statistics of the signal envelope were analyzed by fitting the Rayleigh and GG PDFs to the data. The results showed a significant trend in the goodness of fit of the Rayleigh PDF and the parameters of the GG PDF towards more Rayleigh-like statistics during the peak of apoptosis.

It has previously been demonstrated that other distributions are capable of providing significantly better fits than the
Rayleigh distribution [5], [9], [17]. The result that the GG PDF provided a significantly better fit agrees with previous investigations using high and lower frequency US in tissues.

The trend towards increased goodness of fit during the peak of apoptosis in the mammary tissue provides some indications as to which properties of the tissue are important in determining the signal statistics. The assumptions of the Rayleigh PDF that the scatterers are much smaller than the wavelength and at random positions do not necessarily apply when imaging tissues with HFUS. It is believed that the increased goodness of fit of the Rayleigh PDF during the peak of apoptosis is due to two changes in the tissue, making the scattering interaction closer to Rayleigh statistics. First the reduction in the size of the nucleus, potentially the primary scattering source, during the apoptotic process makes the interaction closer to the assumptions. Second, the restructuring process produces an increase in randomization of the scatterer positions. We are currently performing investigations with suspensions of cells of various sizes that indicate that randomization and number density are both important factors in determining the statistics of the backscatter envelope. It is thus possible that the changes 


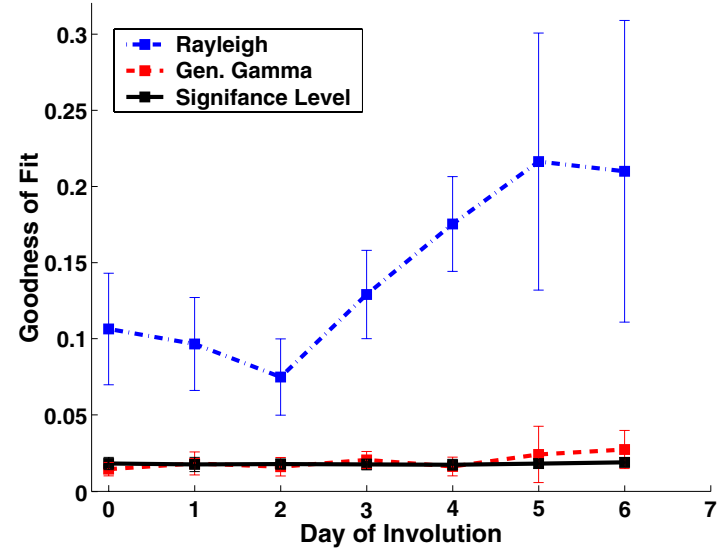

Fig. 3. Kolmogorov-Smirnov goodness of fit values for the fits of the Rayleigh and GG PDFs to HFUS data collected from mouse mammary tissue during involution. Lower KS values represent a better fit to the data. The significant level represents the $95 \%$ confidence level and can be used as a reference to compare the KS values of each day.

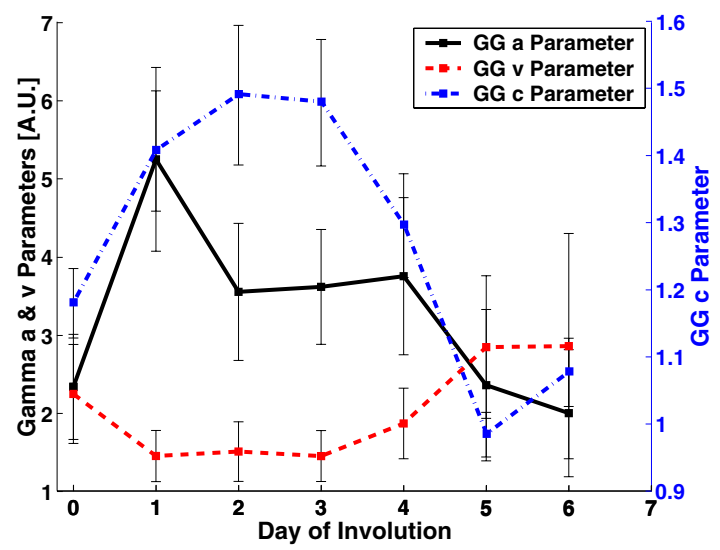

Fig. 4. The GG $a, c$ and $v$ parameters with $95 \%$ confidence intervals for HFUS data from mouse mammary tissue during involution. Data were collected from four mice daily for 7 days. As $c \rightarrow 2$ and $v \rightarrow 1$ the GG PDF becomes the Rayleigh PDF.

observed to tissue structure are responsible for the more Rayleigh-like statistics observed.

\section{CONCLUSION}

The results demonstrate that HFUS signal statistics can be used to monitor tissue restructuring and potentially cell death in vivo. The GG distribution provided a better fit to the HFUS data than the Rayleigh distribution throughout the involution period. There was a significant trend in the goodness of fit of the Rayleigh distribution, indicating that the statistics were most Rayleigh-like between days 2-3 of involution. This corresponded well with the fit parameters of the GG distribution, which approached the special case where it approximates to the Rayleigh distribution.

Mouse mammary tissue exhibits a peak of apoptosis during the restructuring at day 3 of involution. This coincides with the trend towards Rayleigh statistics between days 2-3. This provides an in vivo demonstration that the technique of envelope statistics analysis is capable of monitoring the structural changes that occur during cell death.

\section{ACKNOWLEDGMENT}

The authors would like to acknowledge support from the Canada Foundation for Innovation, the Whitaker Foundation, the Canadian Institutes of Health Research and the Natural Sciences and Engineering Research Council of Canada.

\section{REFERENCES}

[1] M. C. Kolios, G. J. Czarnota, M. Lee, J. W. Hunt, and M. D. Sherar, "Ultrasonic spectral parameter characterization of apoptosis," Ultrasound Med Biol, vol. 28, no. 5, pp. 589-97, 2002.

[2] G. J. Czarnota, M. C. Kolios, H. Vaziri, S. Benchimol, F. P. Ottensmeyer, M. D. Sherar, and J. W. Hunt, "Ultrasonic biomicroscopy of viable, dead and apoptotic cells," Ultrasound Med Biol, vol. 23, no. 6, pp. 961-5, 1997.

[3] G. J. Czarnota, M. C. Kolios, J. Abraham, M. Portnoy, F. P. Ottensmeyer, J. W. Hunt, and M. D. Sherar, "Ultrasound imaging of apoptosis: highresolution non-invasive monitoring of programmed cell death in vitro, in situ and in vivo," Br J Cancer, vol. 81, no. 3, pp. 520-7, 1999.

[4] A. S. Tunis, G. J. Czarnota, A. Giles, M. D. Sherar, J. W. Hunt, and M. C. Kolios, "Monitoring structural changes in cells with high frequency ultrasound signal statistics," Ultrasound Med Biol, Submitted July, 2004.

[5] P. M. Shankar, J. M. Reid, H. Ortega, C. W. Piccoli, and B. B. Goldberg, "Use of non-rayleigh statistics for the identification of tumors in ultrasonic b-scans of the breast," IEEE Trans Med Imaging, vol. 12, no. 4, pp. 687-692, 1993.

[6] P. M. Shankar, V. A. Dumane, T. George, C. W. Piccoli, J. M. Reid, F. Forsberg, and B. B. Goldberg, "Classification of breast masses in ultrasonic b scans using nakagami and k distributions," Phys Med Biol, vol. 48, no. 14, pp. 2229-40, 2003.

[7] X. Hao, C. J. Bruce, C. Pislaru, and J. F. Greenleaf, "Segmenting highfrequency intracardiac ultrasound images of myocardium into infarcted, ischemic, and normal regions," IEEE Trans Med Imaging, vol. 20, no. 12, pp. 1373-83, 2001.

[8] - "Characterization of reperfused infarcted myocardium from highfrequency intracardiac ultrasound imaging using homodyned k distribution," IEEE Trans Ultrason Ferroelectr Freq Control, vol. 49, no. 11, pp. 1530-42, 2002.

[9] B. I. Raju and M. A. Srinivasan, "Statistics of envelope of highfrequency ultrasonic backscatter from human skin in vivo," IEEE Trans Ultrason Ferroelectr Freq Control, vol. 49, no. 7, pp. 871-82, 2002.

[10] C. J. Wilde, C. H. Knight, and D. J. Flint, "Control of milk secretion and apoptosis during mammary involution," J Mammary Gland Biol Neoplasia, vol. 4, no. 2, pp. 129-36, 1999.

[11] J. Strutt, "On the resultant of a large number of vibrations of the same pitch and of arbitrary phase," Phil Mag S5, vol. 10, no. 60, pp. 73-78, 1880.

[12] G. J. Czarnota, "Ultrasound imaging of apoptosis in vivo: Effects of subcellular nuclear morphology and cell membrane morphology," in Proceedings of the 10th Congress of the World Federation for Ultrasound in Medicine and Biology, Montreal, Canada, 2002, p. S117.

[13] J. Beaulieu, R. Vlad, L. Taggart, Y. Heng, A. Giles, M. Sherar, J. Hunt, G. J. Czarnota, and M. Kolios, "High-frequency ultrasound characterization of microcellular components," in Proceedings of the 10th Congress of the World Federation for Ultrasound in Medicine and Biology, Montreal, Canada, 2002, p. S123.

[14] E. Stacy, "A generalization of the gamma distribution," The Annals of Mathematical Statistics, vol. 33, no. 3, pp. 1187-1192, 1962.

[15] P. M. Shankar, "Ultrasonic tissue characterization using a generalized nakagami model," IEEE Trans Ultrason Ferroelectr Freq Control, vol. 48, no. 6, pp. 1716-1720, 2001.

[16] J. K. Patel, C. H. Kapadia, and D. B. Owen, Handbook of statistical distributions, ser. Statistics, textbooks and monographs v 20. New York: M. Dekker, 1976.

[17] V. Dutt and J. F. Greenleaf, "Ultrasound echo envelope analysis using a homodyned $\mathrm{k}$ distribution signal model," Ultrason Imaging, vol. 16, no. 4, pp. 265-87, 1994. 